\title{
Using Sound to Synthesize Covalent Organic Frameworks in Water
}

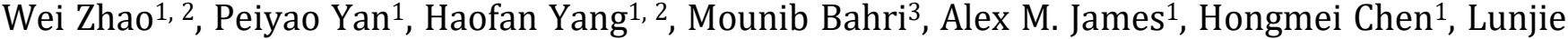 \\ Liu'$^{1}$, Boyu Li1 ${ }^{1}$,Zhongfu Pang1, 2, Rob Clowes ${ }^{1}$, Nigel D. Browning ${ }^{3}$, John W. Ward*1, 2, Yue Wu*1 and \\ Andrew I. Cooper*1, 2
}

\begin{abstract}
Most covalent organic frameworks (COFs) are synthesized using solvothermal conditions ( $>120{ }^{\circ} \mathrm{C},>72 \mathrm{~h}$ ) in harmful organic solvents. We report a strategy for rapidly synthesizing imine-linked COFs ( $<60 \mathrm{~min}$ ) in aqueous acetic acid using sonochemistry, avoiding most of the downsides of solvothermal methods. We first synthesized seven known CoFs using this method and obtained crystallinity and porosity comparable to or better than materials from previously reported solvothermal routes. This sonochemical method even works in highly sustainable solvents, such as food-grade vinegar. The generality of the method was demonstrated by preparing two unreported COFs. Finally, we showed that one sonochemical COF is an excellent photocatalyst for sacrificial hydrogen evolution from water with a more sustained catalytic performance than its solvothermal analog. The speed, ease and generality of this sonochemical method with no sacrifice in material quality makes it an enabling methodology for rapid discovery of new functional COF materials.
\end{abstract}

Crystalline covalent organic frameworks (COFs) have received much attention because of their potential applications in catalysis, adsorption, separations, chemo-sensing, drug delivery, and energy storage and production. ${ }^{1-3}$ The dominant route to COFs is solvothermal synthesis, ${ }^{4}$ but this often requires the use of sealed, pressurized tubes, elevated reaction temperatures $\left(120-200{ }^{\circ} \mathrm{C}\right)$, long reaction times (2-7 days) and toxic organic solvents. This creates a significant incentive to develop alternative methods to synthesize COFs; for example, to accelerate the discovery of new functional materials.

There are alternatives to solvothermal syntheses, such as microwave synthesis ${ }^{5}$ and room-temperature syntheses that involve catalysts. ${ }^{6,7}$ These routes can have reduced reaction times of 1-2 hours or so; that is, much faster than more traditional solvothermal syntheses. However, it is still desirable to avoid the use of toxic organic solvents and metal catalysts, and to accelerate this chemistry further. Solid-state synthesis is one route that eliminates bulk solvent use and reduces waste generation. $p$-Toluenesulfonic acid (PTSA), a strong solid acid, was first used as the catalyst for solid-state COF synthesis by Kandambeth et al. in $2017 .{ }^{8}$ However, a large quantity of PTSA ( $\sim 6$ molar equivalents based on the amine monomers) was required during the synthesis, and high temperature treatment $\left(90-170{ }^{\circ} \mathrm{C}\right)$ for 1 minute to 2 days was needed to obtain the crystalline COFs. ${ }^{9,10}$ Mechanochemical synthesis is another promising solid-state route. The first examples of COF mechanosynthesis were reported by Biswal et al. ${ }^{11}$ Solvent-free mechanochemical processes offer the potential for larger-scale COF synthesis but such studies are rare so far and the COFs so produced show limited crystallinity and porosity. ${ }^{12}$ For example, the mechanically synthesized COFs, TpPa- 1 , TpPa-2, and TpBD, had only moderate crystallinity and low Brunauer-Emmet-Teller (BET) surface areas $\left(61 \mathrm{~m}^{2} \mathrm{~g}^{-1}\right.$ for TpPa-1, $56 \mathrm{~m}^{2} \mathrm{~g}^{-1}$ for TpPa-2, and $35 \mathrm{~m}^{2} \mathrm{~g}^{-1}$ for TpBD) compared to their solvothermal analogs. ${ }^{11}$ Recent work by Emmerling et al. ${ }^{13}$ showed that alternative activation methods such as supercritical $\mathrm{CO}_{2}$ drying ${ }^{14,15}$ may allow access to the porosity of mechanochemically prepared COFs.

Aqueous COF synthesis is attractive because it avoids the use of hazardous organic solvents. There are a limited number of reports of keto-enamine-based and azine-linked COFs produced using hydrothermal syntheses, ${ }^{16,17}$ but long reaction times (0.5-3 days) and elevated temperatures $\left(>120^{\circ} \mathrm{C}\right)$ were still required in the examples reported so far. Moreover, the authors concluded that their method only works well for COFs that are stabilized by keto-enol tautomerism, since conventional imine bonds are susceptible to the reversible back reaction upon exposure to water. Recently, Martín-Illán et al. developed a protocol for the aqueous synthesis of imine-linked COFs at $80{ }^{\circ} \mathrm{C}$ for 5 days. ${ }^{18}$ However, a very low concentration of starting monomers was required due to their low aqueous solubility and an organic solvent (DMSO) was used to aid solubilization in some cases. Even when microwave radiation was used to accelerate the chemistry, a 5-hour reaction time was still needed to obtain the COF.

Sonochemistry refers to reactions that are driven by high-energy ultrasound. In sonochemistry, acoustic cavitation occurs because of the formation, growth, and implosive collapse of bubbles in a liquid due to ultrasonic shearing. This produces localized hot spots that can accelerate reactions. ${ }^{19}$ One advantage of sonochemistry is that the equipment required-an ultrasonic probe (Figure 1)-is compact, easy to use, relatively inexpensive (around the cost of a basic rotary evaporator) and particularly amenable to small-scale discovery chemistry. To our knowledge, the only reports of sonochemical COF synthesis are by Yang and co-workers ${ }^{20}$ and by Yoo et $a .^{21}$ In those studies, first-generation COFs based on the less stable boroxine chemistry were prepared. Here we focus on the stable, functional COFs synthesized via imine condensation that are the focus of much current research.

\footnotetext{
${ }^{1}$ Materials Innovation Factory and Department of Chemistry, University of Liverpool, Crown Street, Liverpool L69 7ZD, UK; ${ }^{2}$ Leverhulme Research Centre for Functional Materials Design, Materials Innovation Factory and Department of Chemistry, University of Liverpool, Liverpool, UK; ${ }^{3}$ Albert Crewe Centre for Electron Microscopy, University of Liverpool, Liverpool, L69 3GL, UK.
} 

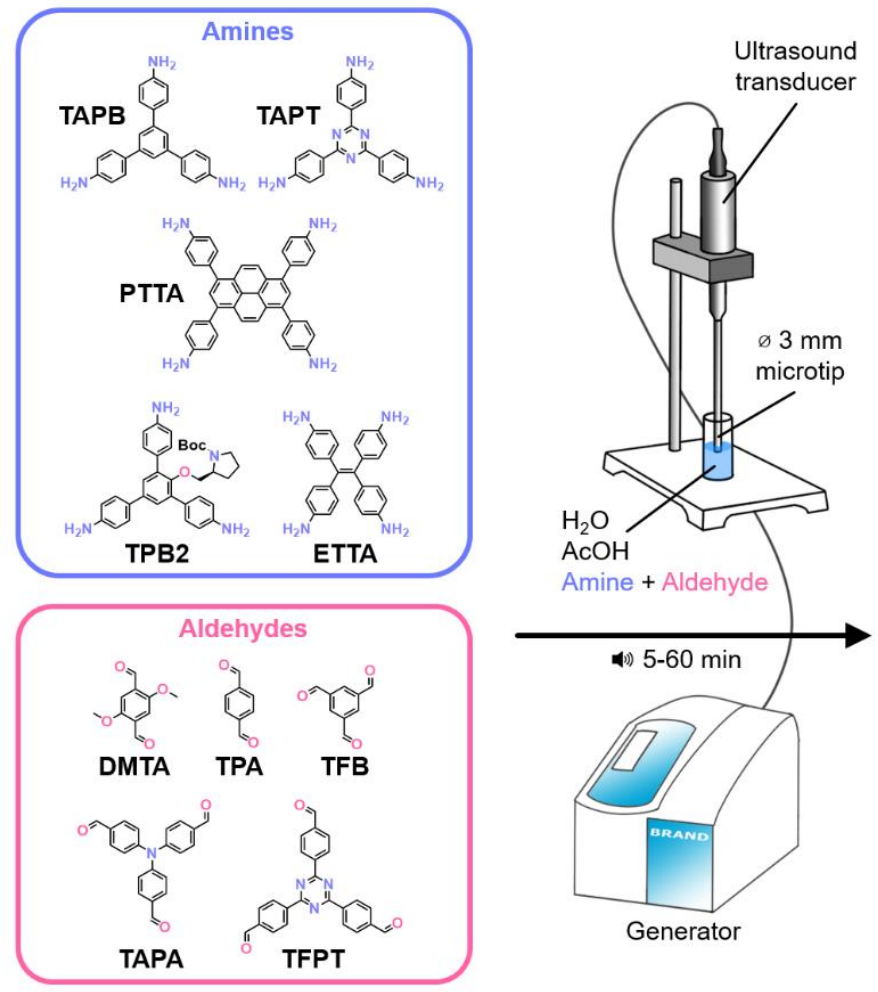
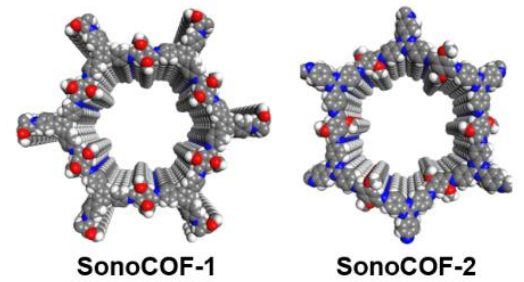

SonoCOF-2

TAPT-DMTA

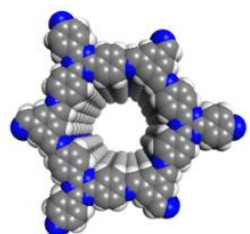

SonoCOF-4 TAPT-TFPA
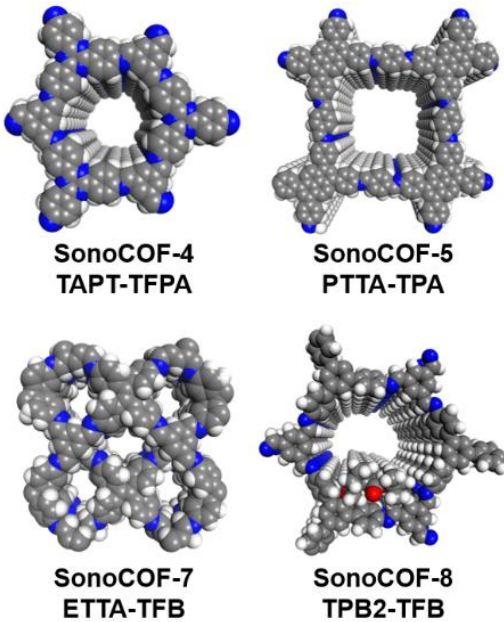

SonoCOF-8 TPB2-TFB

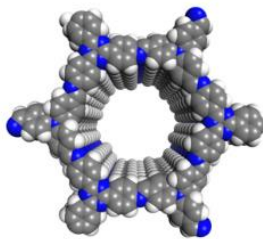

SonoCOF-3

TAPT-TFPA

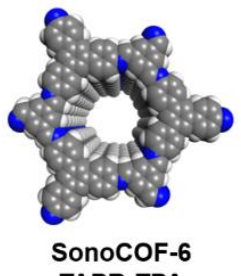

TAPB-TPA

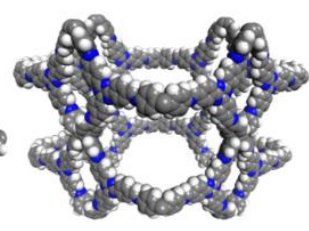

SonoCOF-9 ETTA-TFPT

Figure 1. Apparatus and conditions used for sonochemical synthesis, the COFs studied, and the monomers used to make them.

We present a strategy for the aqueous sonochemical synthesis of imine-linked COFs that allows very short reaction times without the use of any organic solvents, while often yielding materials with properties that are superior to those obtained using other synthetic procedures. To thoroughly test this approach, we report the preparation of seven known COFs ${ }^{22-27}$ and two new COFs. These 'sonoCOFs' are prepared in less than 1 hour with high crystallinity and porosity levels that are comparable to or better than COFs formed by solvothermal methods. Hazardous organic solvents are avoided, making the method safer, cleaner, and more economical. Moreover, this method is simple and efficient compared to conventional solvothermal methods. We also tested the functional performance of the sonoCOFs against solvothermal COFs for sacrificial photocatalytic $\mathrm{H}_{2}$ evolution. Several COFs prepared by the sonochemical method had excellent photocatalytic performance; in particular, sonoCOF-3 showed better sustained hydrogen evolution over 40 hours than its solvothermal analog.

\section{Results and discussion}

Discovery and systematic investigation of the sonochemical synthesis of a prototypical COF. To test our initial hypothesis that sonochemical synthesis might be feasible for imine-linked COFs, we chose a prototypical imine-linked COF known as "TAPB-DMTA COF", formed from the condensation of 1,3,5-tris(4-aminophenyl)benzene (TAPB) and 2,5-dimethoxybenzene-1,4-dicarboxaldehyde (DMTA), known for its stability and crystallinity. ${ }^{22}$ We discovered that this COF was easily synthesized using sonochemistry in $6 \mathrm{M}$ aqueous acetic acid $(\mathrm{AcOH})$ without the need for organic solvents. We therefore selected this COF (Figure 2a, referred to here as sonoCOF-1) as a model system to explore the synthetic conditions in more detail. All of the sonochemical reactions were performed with a $550 \mathrm{~W}$ ultrasonic probe in continuous mode at $50 \%$ sonication power using a $3 \mathrm{~mm}$ microtip probe.

We first investigated the effect of reaction time. Reactions were run for 5, 10, 30 and 60 min. The formation of sonoCOF-1 was confirmed by powder X-ray diffraction (PXRD) and Fourier-transform infrared (FT-IR) spectroscopy (Figure 2b and 2d). SonoCOF1 was produced in $38 \%$ yield after just 5 minutes sonication. Yields increased rapidly with reaction time: $76 \%$ for 10 min, $93 \%$ for $30 \mathrm{~min}$ and $93 \%$ for 60 min (Figure 2e).

The permanent porosity of sonoCOF-1 was assessed by nitrogen adsorption isotherms measured at $77 \mathrm{~K}$. As shown in Figure $2 \mathrm{c}$ and 2e, longer sonication times led to an increase in both the BET surface area and the total pore volume, reaching a maximum of $2059 \mathrm{~m}^{2} \mathrm{~g}^{-1}$ and $1.80 \mathrm{~cm}^{3} \mathrm{~g}^{-1}$, respectively, after 60 minutes (insufficient sample was obtained for reliable measurements from the 5 minute reaction). Longer reactions times (90 and $120 \mathrm{~min}$ ) showed little further change (Figure S42). Among the conditions tested, 1 hour was found to be the optimal reaction time for the formation of sonoCOF-1, which much faster than the 72 hours commonly used for the solvothermal reaction. ${ }^{22}$

We also studied the effect of AcOH concentration on the formation of sonoCOF-1. PXRD analysis (Figure S20) showed that crystalline sonoCOF-1 could also be formed in $1 \mathrm{M}$ and $3 \mathrm{M}$ aqueous acetic acid, but lower BET surface areas $\left(261 \mathrm{~m}^{2} \mathrm{~g}^{-1}\right.$ for $1 \mathrm{M}$ and $1392 \mathrm{~m}^{2} \mathrm{~g}$ 1 for $3 \mathrm{M})$ and pore volumes $\left(0.22 \mathrm{~cm}^{3} \mathrm{~g}^{-1}\right.$ for $1 \mathrm{M}$ and $1.11 \mathrm{~cm}^{3} \mathrm{~g}^{-1}$ for $\left.3 \mathrm{M}\right)$ were obtained. SonoCOF-1 could even be prepared using food-grade distilled vinegar $(\sim 1 \mathrm{M} \mathrm{AcOH})$ giving a BET surface area of $380 \mathrm{~m}^{2} \mathrm{~g}^{-1}$ and pore volume of $0.32 \mathrm{~cm}^{3} \mathrm{~g}^{-1}$. 

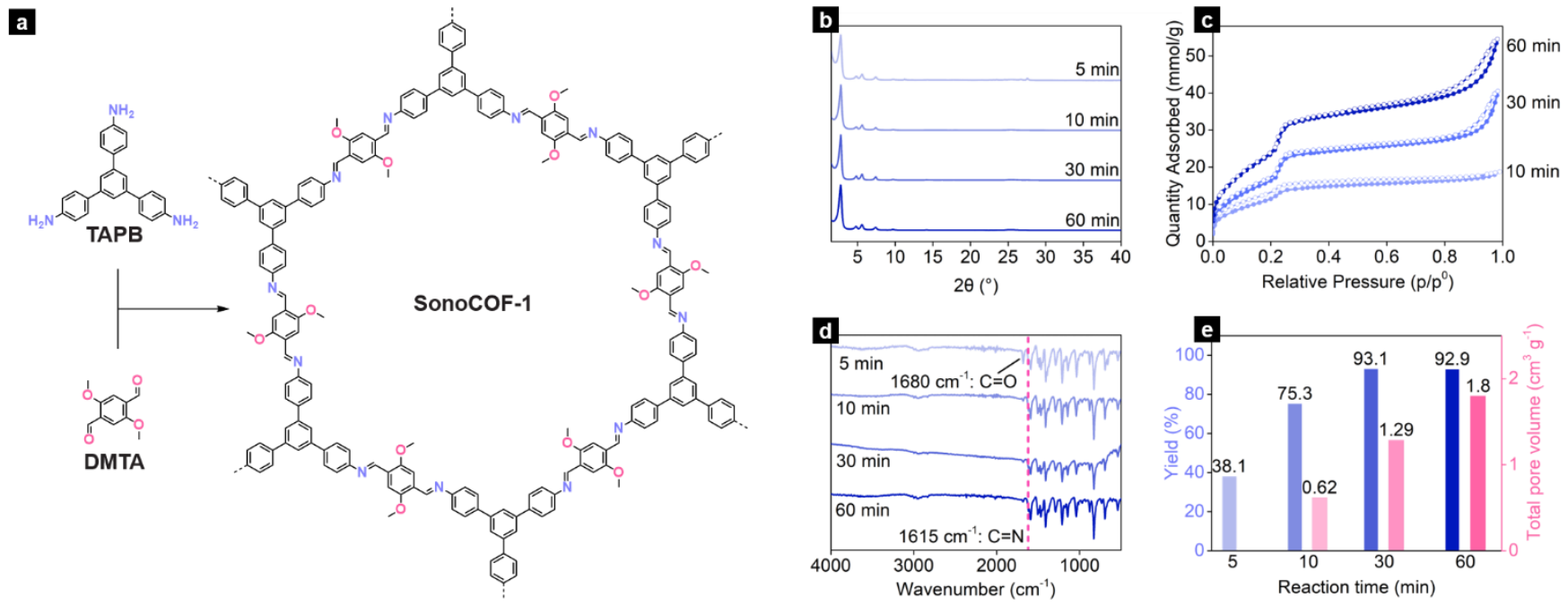

Figure 2. (a) Sonochemical synthesis route for sonoCOF-1. (b) PXRD, (c), N2 adsorption, (d) FT-IR spectra, and (e) yields (blue bars) and total pore volumes (pink bars) of sonoCOF-1 prepared under different reaction times.

Scope of the aqueous sonochemical method. To demonstrate the generality of our aqueous sonochemical strategy, we synthesized a diverse set of 6 more known COFs that were prepared previously using solvothermal methods: sonoCOF-2 ('COF-JLU5') ${ }^{23}$ composed of 4,4',4"'-(1,3,5-triazine-2,4,6-triyl)trianiline (TAPT) and DMTA, sonoCOF-3 ('TPT-COF-4') ${ }^{27}$ composed of TAPT and tris(4formylphenyl)amine (TFPA), sonoCOF-4 ('N3-COF') 25 composed of TAPT and 1,3,5-triformylbenzene (TFB), sonoCOF-5 ('Py-1P $\left.\mathrm{COF}^{\prime}\right)^{28}$ composed of 4,4',4",4"'-(pyrene-1,3,6,8-tetrayl)tetraaniline (PTTA) and terephthalaldehyde (TPA), sonoCOF-6 ('RT-COF$\left.1^{\prime}\right)^{29}$ composed of TAPB and TPA, and sonoCOF-7 ('COF-432') ${ }^{24}$ composed of 4,4',4',4'"-(ethene-1,1,2,2-tetrayl)tetraaniline (ETTA) and TFB (Table S1). The corresponding sonoCOF-x (os, 'organic solvent', $x=2-7$ ) series was also synthesized in mixtures of $1,4-$ dioxane/mesitylene or n-BuOH/o-DCB as a control (details in ESI section 2.2). The identities of the sonoCOF reaction products were confirmed by elemental analysis, FT-IR spectroscopy, thermogravimetric analysis (TGA), PXRD, and $\mathrm{N}_{2}$ sorption analysis. 
a

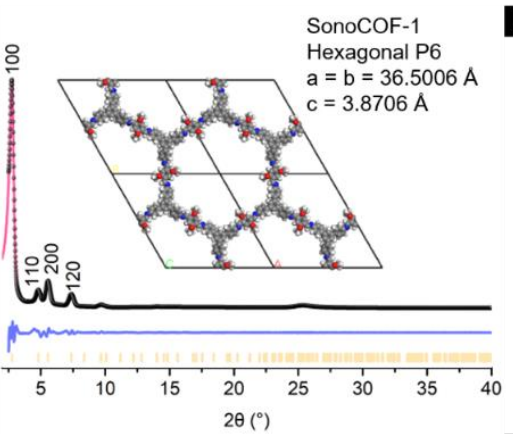

d

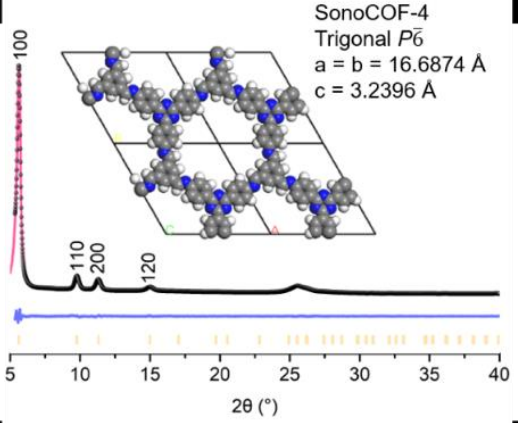

g

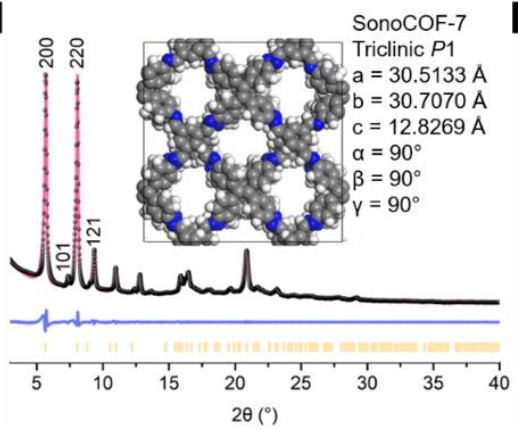

b

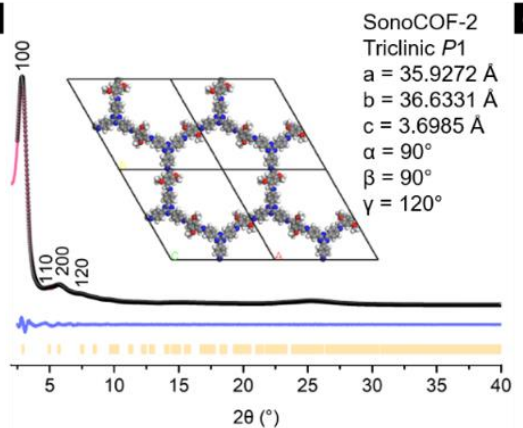

e

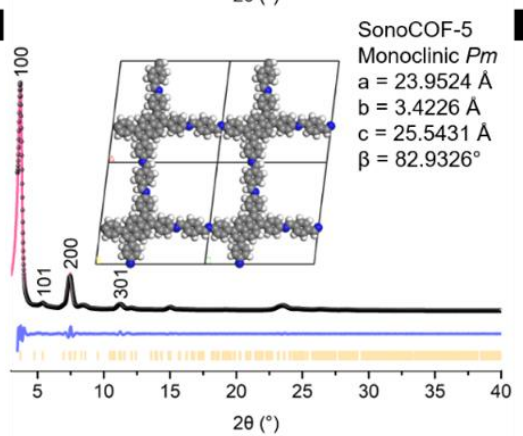

h

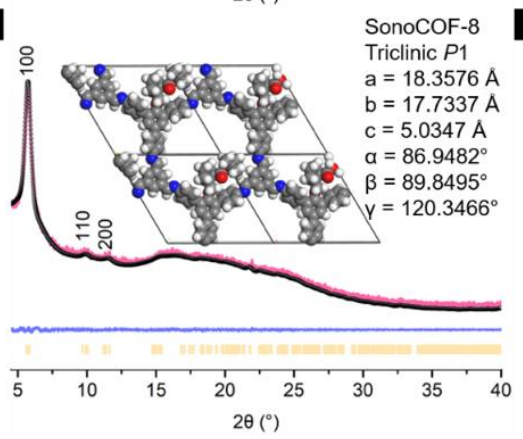

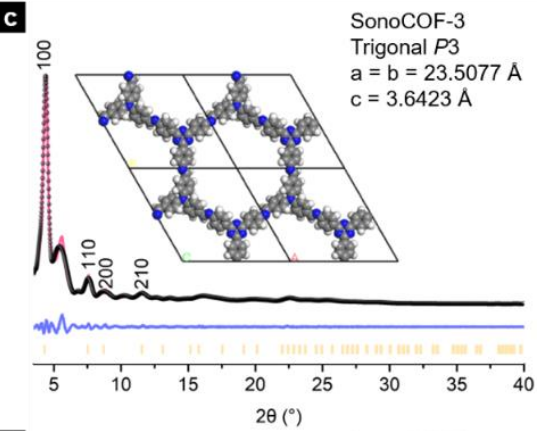
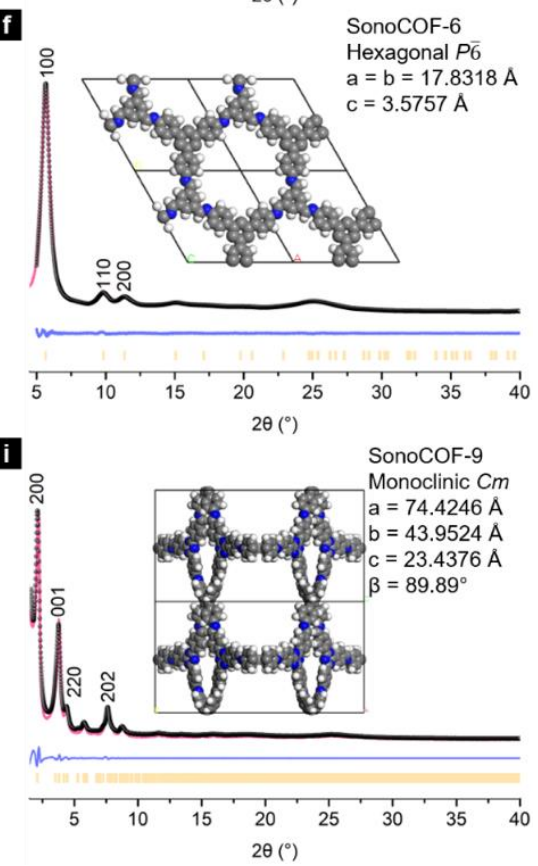

Figure 3. PXRD patterns of (a-i) sonoCOF-1-9. Pink lines: yobs, experimental PXRD data; black dots: ycalc, Pawley refinement profile; blue lines: $y_{\text {obs }}-\mathrm{y}_{\text {calc, }}$ residual; yellow marks: hkl positions calculated for that phase. Insets: Modelled crystal structures. C, gray; $\mathrm{H}$, white; N, blue; 0 , red.

Table 1. Comparison of total pore volumes and BET surface areas of sonoCOFs (reaction time: 60 min; reaction temperature: started at ambient temperature, $\sim 20^{\circ} \mathrm{C}$, stabilizing at $\sim 78{ }^{\circ} \mathrm{C}$ after 10 minutes sonication, Figure S106) with equivalent materials reported in the literature prepared under solvothermal conditions $\left(120^{\circ} \mathrm{C}\right.$ for $3-7$ days). a Pore volume not reported. b Pore volume is calculated at $\mathrm{P} / \mathrm{P}^{0}=0.97$. ${ }^{\mathrm{c}} \mathrm{BET}$ surface areas are calculated under $\mathrm{P} / \mathrm{P}^{0}=0.01-0.2$ (see Supporting Information, Section 3.7).

\begin{tabular}{|c|c|c|c|c|c|}
\hline SonoCOFs & Pore vol. $\left(\mathrm{cm}^{3} \mathrm{~g}^{-1}\right)$ & This work $\left(\mathrm{cm}^{3} \mathrm{~g}^{-1}\right)^{\mathrm{b}}$ & $\mathrm{S}_{\text {BET }}\left(\mathrm{m}^{2} \mathrm{~g}^{-1}\right)$ & This work $\left(\mathrm{m}^{2} \mathrm{~g}^{-1}\right)^{\mathrm{c}}$ & References \\
\hline \multirow{2}{*}{ SonoCOF-1 } & 1.28 & \multirow{2}{*}{1.80} & 2105 & \multirow{2}{*}{2059} & 22 \\
\hline & 1.28 & & 1927 & & 30 \\
\hline \multirow{3}{*}{ SonoCOF-2 } & 1.59 & & 1632 & \multirow{3}{*}{1890} & 23 \\
\hline & $-\mathrm{a}$ & 1.45 & 1314 & & 31 \\
\hline & 1.15 & & 1036 & & 32 \\
\hline SonoCOF-3 & $-\mathrm{a}$ & 1.04 & 1132 & 1587 & 27 \\
\hline \multirow{4}{*}{ SonoCOF-4 } & 0.56 & \multirow{4}{*}{0.67} & 1142 & \multirow{4}{*}{1425} & 25 \\
\hline & 0.66 & & 1000 & & 33 \\
\hline & 0.55 & & 1149 & & 34 \\
\hline & 0.55 & & 1163 & & 30 \\
\hline \multirow{3}{*}{ SonoCOF-5 } & 1.09 & \multirow{3}{*}{1.51} & 2210 & \multirow{3}{*}{1746} & 28 \\
\hline & $-\mathrm{a}$ & & 1520 & & 26 \\
\hline & 1.25 & & 2039 & & 35 \\
\hline \multirow{5}{*}{ SonoCOF-6 } & $-a$ & \multirow{5}{*}{0.81} & 1268 & \multirow{5}{*}{1013} & 36 \\
\hline & $-a$ & & 687 & & 37 \\
\hline & $-\mathrm{a}$ & & 888 & & 38 \\
\hline & $-\mathrm{a}$ & & 420 & & 39 \\
\hline & 0.44 & & 435 & & 34 \\
\hline SonoCOF-7 & 0.43 & 0.54 & 895 & 940 & 24 \\
\hline
\end{tabular}


All sonoCOFs exhibited good crystallinities as gauged by PXRD. By contrast, not all sonoCOFs could be prepared as crystalline products in the organic solvents we tested; for example, sonoCOF-3 (os) and sonoCOF-7 (os) were almost amorphous (Figure S21). These results suggest that the aqueous sonochemical synthesis of imine COFs may be more robust than equivalent syntheses in organic solvents. As shown in Figure 3, the experimental PXRD patterns of sonoCOF-1-7 are consistent with the structures reported in the literature. The unit cell parameters of sonoCOF-1-7 were refined by the Pawley method (Table S3-S9). We note that sonoCOF-3 shows an unassigned peak at around $5.6^{\circ}$, which was also observed in other reports ${ }^{27,40}$ - this is thought to be from stacking faults. Scanning electron microscopy (SEM) showed that the sonoCOF samples comprised sub-micron crystallites (Figure S23-40).

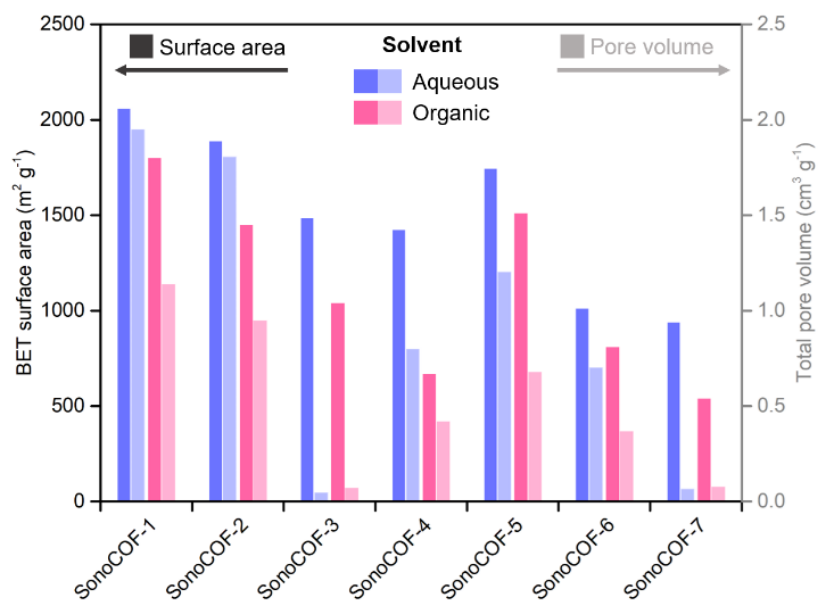

Figure 4. Comparison of BET surface areas and total pore volumes of sonoCOFs synthesized in aqueous (blue bars) and organic solvents (pink bars).

The permanent porosity of the sonoCOFs was assessed by nitrogen adsorption-desorption isotherms measured at $77 \mathrm{~K}$ after degassing at $120{ }^{\circ} \mathrm{C}$ for $12 \mathrm{~h}$. For sonoCOF-1 and sonoCOF-2, the adsorption isotherms show characteristic type IV features (Figure S41a and S41b) with BET surface areas of 2059 and $1890 \mathrm{~m}^{2} \mathrm{~g}^{-1}$, respectively. For sonoCOF-3-7, the adsorption isotherms showed a rapid uptake at a low relative pressure of $\mathrm{P} / \mathrm{P}^{0}<0.01$ and reached saturation at $\mathrm{P} / \mathrm{P}^{0}<0.1$, which is characteristic of a type $\mathrm{I}$ isotherm for a microporous solid (Figure S41c-S41g). All sonoCOFs showed some hysteresis at higher pressures, likely due to the inter-particle mesoporosity formed from the aggregation of the COF nano-particles. Application of density functional theory (DFT) showed the pore size distribution of sonoCOF-1-7 to be centered at $\sim 3.17,2.95,1.84,1.34,2.40,1.49$ and 1.52 nm, respectively. The porosity of the sonoCOF (os) materials was measured under the same conditions as a control. All sonoCOFs exhibited higher BET surface areas and total pore volumes than their sonoCOF (os) analogs (Figure 4).

Comparison of literature BET surface areas and total pore volumes for the solvothermal analogs of these sonoCOFs showed similar or higher values for the sonoCOFs (Table 1) with the exception of just one example (sonoCOF-5, reference 28 and 35 ). We note here that BET surface areas are sensitive to the relative pressure range used for the calculation. Also, the increase in total pore volume might be ascribed, at least in part, to inter-particle gas condensation for some of the sonoCOFs (e.g., sonoCOF-3, see Figure S103), which typically comprise smaller microparticles than the analogous solvothermal materials.

Investigation into sonochemical COF formation. We next performed a series of control experiments to show that it is indeed sound energy that is driving these reactions. Reaction mixtures of the same composition used for sonoCOF-1-7 were treated under three different conditions:

(i) The amine and aldehyde monomers were added to an aqueous solution of acetic acid in a 4 mL vial and left at room temperature for 1 hour without stirring or sonication (Control-1).

(ii) The same reagents were sonicated in aqueous acetic acid solution using a much less powerful standard laboratory ultrasonic cleaning bath at $25-38^{\circ} \mathrm{C}$ for 1 hour, as opposed to using an ultrasonic probe (Control-2).

(iii) The same reagents were stirred in an aqueous acetic acid solution at $80{ }^{\circ} \mathrm{C}$ in a heating block for 1 hour (Control-3).

In Control-1, COFs were formed with significantly lower crystallinity and yield, except sonoCOF-2 and sonoCOF-3, which did not form at all under these conditions. The Control-2 and Control-3 conditions were more favorable, but again, products showed lower surface areas, lower total pore volumes and lower yields in all but one case. Notably, sonoCOF-3 did not form at all under any of the control conditions. These results are summarized in Table S1.

While sonocrystallization is known to lead to crystals with better uniformity, regularity, and less agglomeration in some cases, the mechanisms for these effects remain contentious because it is difficult to probe sonochemical processes on the time and length scales at which they occur. ${ }^{41}$ Performing the reaction in aqueous acetic acid seems to be part of the reason for the high crystallinity and 
porosity observed, since the analogous reactions in organic solvent/acetic acid mixtures were, in general, less successful. Certainly, water plays an important role during imine COF formation and several other reports have shown that it aids COF crystallization. ${ }^{42-44}$ We note that the amine monomers were found to be highly soluble under these conditions due to the protonation with acetic acid. It is also possible that protonation modulates the reactivity.

Our results do not agree with the conclusions of Thote et al. ${ }^{16}$ and Martín-Illán et al. ${ }^{18}$ who suggest that it is not possible to obtain imine-based COFs in high concentration aqueous AcOH solutions due to the protonation of the amine monomers. Our results clearly show that COFs can indeed be formed under such conditions, at least when using sonochemistry.

Discovery of new COFs. Having developed a robust method for the synthesis of seven known 2D COFs, we next attempted the synthesis of two unreported COFs: a 2D COF with pendant functionalization, and a COF with 3D connectivity (Figure 5). SonoCOF-8 is constructed from a chirally-functionalized building block TPB2, ${ }^{45}$ while sonoCOF-9 is a 3D COF based on the substitution of TFPB with TFPT in the family of 3D ETTA-containing COFs. ${ }^{46}$ Formation of sonoCOF-8 and sonoCOF-9 was corroborated by FT-IR and CPMAS ${ }^{13} \mathrm{C}$ NMR spectroscopies (see Supporting Information, section 3.2 and 3.8).
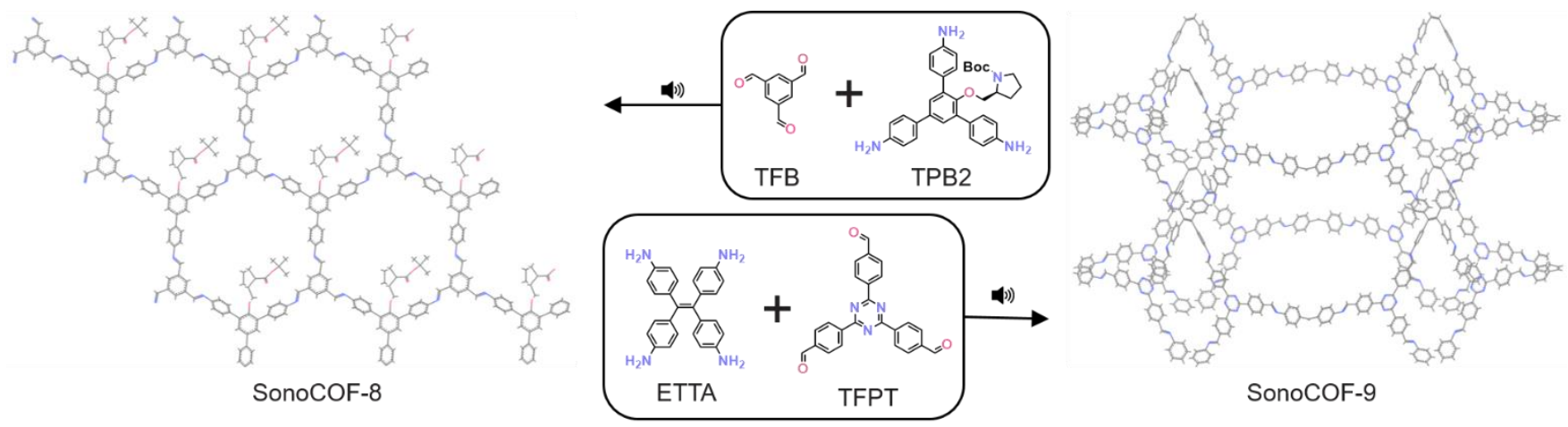

Figure 5. Synthetic routes for sonoCOF-8 and sonoCOF-9.

PXRD measurements for sonoCOF-8 (Figure 3h) and sonoCOF-9 (Figure 3i) showed diffraction peaks that were consistent with their simulated structures. The experimental PXRD pattern for sonoCOF-8 matched well with a simulated eclipsed AA-stacking arrangement (Figure S17). SonoCOF-9 exhibits good crystallinity with intense and sharp low-angle reflections, displaying a diffraction pattern very similar to the isostructural 3D-ETTA-TFPB COF. ${ }^{46}$ However, rather than the previously reported phase 3D-ETTA-TFPB COF, we found that our diffraction data matched better with a monoclinic structure that we simulated with the ffc net (Figure S18). The crystal structure of sonoCOF-9 was assigned to the space group $C m$ with unit cell parameters of $a=74.42 \AA, b=43.95 \AA, c=23.44 \AA$, $\beta=89.89^{\circ}$.

Both sonoCOFs possessed permanent porosity, as shown by nitrogen adsorption-desorption isotherms measured at $77 \mathrm{~K}$ (Figure S39). The BET surface area was calculated to be $154 \mathrm{~m}^{2} \mathrm{~g}^{-1}$ for sonoCOF-8 and $984 \mathrm{~m}^{2} \mathrm{~g}^{-1}$ for sonoCOF-9, with total pore volumes of 0.19 and $0.52 \mathrm{~cm}^{3} \mathrm{~g}^{-1}$ at $\mathrm{P} / \mathrm{P}^{0}=0.97$ for sonoCOF-8 and sonoCOF-9, respectively.

Photocatalytic $\mathbf{H}_{2}$ evolution. Recently, COFs have prompted interest as heterogeneous catalysts for photocatalytic water splitting. ${ }^{47}$ Since high crystallinity is thought to be favourable for photocatalytic performance, ${ }^{47,48}$ we tested these sonoCOFs for sacrificial photocatalytic $\mathrm{H}_{2}$ evolution.

Initially, the photocatalytic activities of the sonoCOFs were screened using a high throughput instrument (see Supporting Information Section 1). As shown in Figure 6A, sonoCOF-3, sonoCOF-6 and sonoCOF-9 exhibited high efficiency for photocatalytic hydrogen evolution with ascorbic acid as the sacrificial electron donor. 


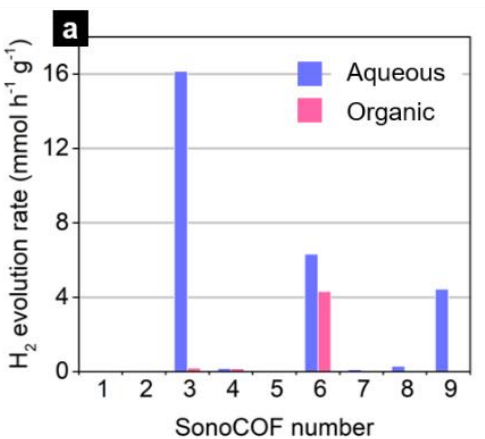

d
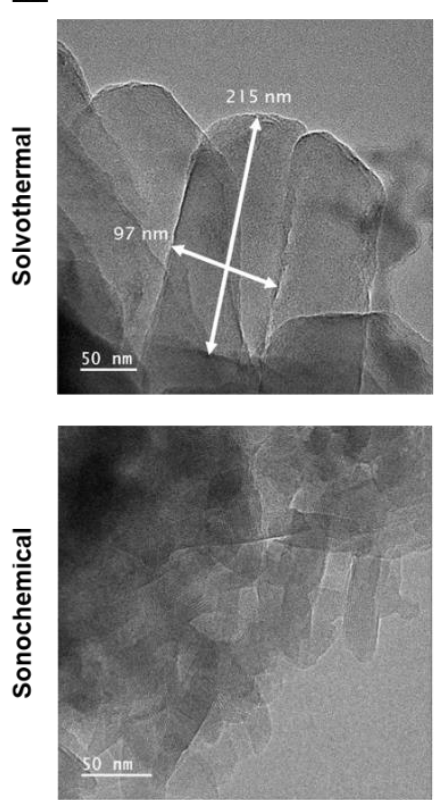

b

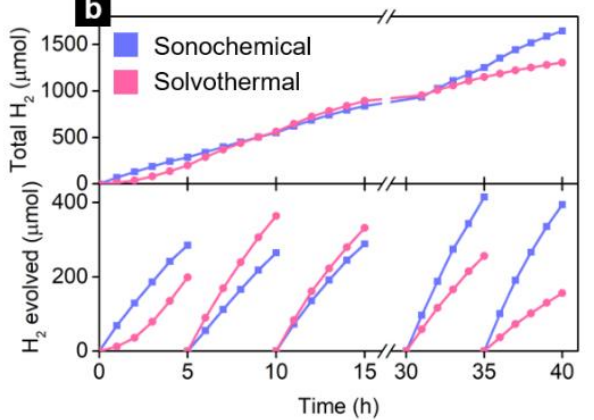

Post-photocatalysis
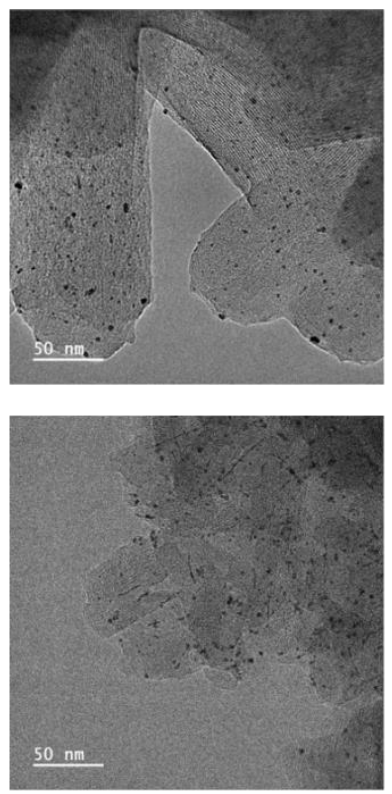

c

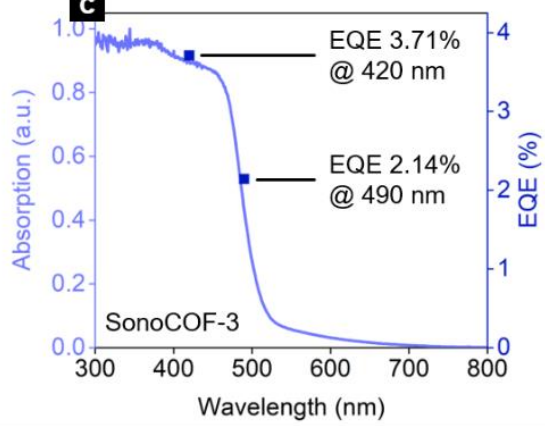

e

HRTEM of pristine sonoCOF-3
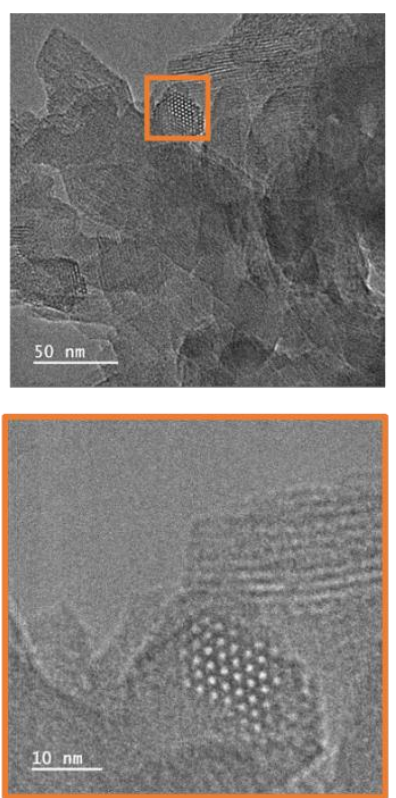

Figure 6. (a) Screening of sonoCOFs for sacrificial photocatalytic hydrogen evolution. Conditions: $2 \mathrm{mg}$ of $\mathrm{COF}$ catalyst, $16 \mu \mathrm{L} \mathrm{H}_{2} \mathrm{PtCl} 6$ solution ( $0.8 \mathrm{wt} \%$ in water), $5 \mathrm{~mL} 0.1 \mathrm{M}$ ascorbic acid (AA) as a sacrificial electron donor, $2 \mathrm{~h}$ solar simulator illumination. Blue bars = aqueous sonoCOFs; pink bars = organic sonoCOFs (os). (b) Plot showing sacrificial photocatalytic hydrogen evolution versus time for sonoCOF-3 and solvoCOF-3 over $40 \mathrm{~h}$ ( $5 \mathrm{mg}$ catalyst in water, diluted $\mathrm{H}_{2} \mathrm{PtCl}_{6}$ solution as a platinum precursor (4 wt $\%$ loading), $0.1 \mathrm{M}$ ascorbic acid $(25 \mathrm{ml}), \lambda>420 \mathrm{~nm}$ ). The sample was degassed after 5 and $10 \mathrm{~h}$ to prevent saturation of the detector, then left under continuous illumination for $15 \mathrm{~h}$ and again degassed after $30 \mathrm{~h}$ and $35 \mathrm{~h}$. After $30 \mathrm{~h}, 1.25 \mathrm{mmol}$ of ascorbic acid was added. (c) Overlay of the UV/vis absorption spectrum of sonoCOF-3 with external quantum efficiency (EQE) for the photocatalytic hydrogen evolution reaction with sonoCOF-3 at two different incident light wavelengths. (d) TEM images of solvoCOF-3 and sonoCOF-3 before and after photocatalytic testing and (e) HRTEM image of sonoCOF-3 clearly showing the hexagonal pore structure of a single crystallite.

The best performing catalyst, sonoCOF-3 was studied in more detail. In the presence of ascorbic acid as a sacrificial electron donor, with $\mathrm{Pt}$ as a co-catalyst, sonoCOF-3 gave $\mathrm{H}_{2}$ evolution rates of up to $16.6 \mathrm{mmol} \mathrm{h}^{-1} \mathrm{~g}^{-1}$ under visible light $(\lambda>420 \mathrm{~nm}, 300 \mathrm{~W}$ Xenon lamp) (Figure 6B). This is one of the highest sacrificial hydrogen evolution rates reported for a COF (Table S13). An external quantum efficiency (EQE) of $3.71 \%$ was determined for sonoCOF-3 at $420 \mathrm{~nm}$ (Figure 6c); for comparison, $3.2 \%$ was reported for FS-COF 47 at $420 \mathrm{~nm}$ and $0.44 \%$ for $\mathrm{N}_{3}-\mathrm{COF}^{49}$ at $450 \mathrm{~nm}$. We also compared the photocatalytic performance of sonoCOF-3 with its solvothermal analog (solvoCOF-3) in longer-term photocatalysis experiments under visible light irradiation $(\lambda>420 \mathrm{~nm})(\mathrm{Figure} 6 \mathrm{~b})$. Both COFs continue to show hydrogen evolution for 40 hours, but sonoCOF-3 maintains very high activity at the end of the run while the catalytic efficiency of solvoCOF-3 is reduced. The PXRD pattern for sonoCOF-3 catalyst showed that crystallinity was retained after longterm irradiation to a greater extent than for solvoCOF-3 (Figure S98). In the course of this study, Thomas et al.40 also reported high $\mathrm{H}_{2}$ evolution rates using a COF (TtaTfa) with the same chemical structure as sonoCOF-3. TfaTfa was synthesized using solvothermal conditions and the photocatalytic experiments were conducted under similar conditions. Degradation of catalytic activity and loss of crystallinity over longer-term photocatalysis was also observed for TtaTfa.

TEM images (Figure 7) show that both sonoCOF-3 and solvoCOF-3 form rectangular particles, but the sonoCOF-3 particles are around half the length and width $(\sim 50 \times 100 \mathrm{~nm}$ vs $\sim 100 \times 200 \mathrm{~nm})$. Despite their smaller size, the sonoCOF-3 crystallites are well defined 
and highly crystalline, as evidenced by the HRTEM images. It is possible that the high crystallinity of the sonoCOF-3 particles enhances their resistance to degradation, which is one possible explanation for their sustained hydrogen production. SonoCOF-3 also exhibits better dispersibility in aqueous media (Figure S104), which is known to favor photocatalytic activity for materials of this type. ${ }^{50}$ We also observed over several TEM images that the Pt co-catalyst appeared to be better dispersed on the sonoCOF-3 particles (see also Figure S111), and improved resistance to Pt agglomeration will tend to enhance photocatalytic performance, too.

\section{Conclusion}

In summary, we present a rapid and clean method for COF synthesis using sonochemistry. Seven reported COFs and two new COFs were prepared. These materials were obtained in just 60 minutes by simply mixing and sonicating the reagents in water with acetic acid, and the products showed high porosity and high crystallinity. The temperature of the solutions increased during sonication, but control experiments with simple heating (Control 3; Table S1) showed that the materials properties obtained are superior under sonication conditions. These sonochemical methods are more facile than conventional solvothermal syntheses, and this fast and convenient method is particularly suited to high-throughput discovery approaches. Aqueous sonochemical synthesis has several advantages: the cost and toxicity of the process is reduced, and the need for solvent screening to optimize crystallinity is avoided. The functional properties of COFs can also be improved through aqueous sonochemical routes, both in terms of porosity levels and other functional properties, such as photocatalytic activity. Moreover, the low reaction temperatures broaden the range of COF monomers that can be considered to encompass functionality that is thermally less stable, providing that is that these monomers can tolerate acidic conditions. We believe that sonochemical synthesis could be a powerful new tool for COF researchers and that it deserves broader investigation for other functional materials, such as porous amorphous organic polymers, conjugated microporous polymers, or perhaps porous organic cages.

\section{Online content}

Any methods, additional references, Nature Research reporting summaries, source data, extended data, supplementary information, acknowledgements, peer review information; details of author contributions and competing interests; and statements of data and code availability are available at...

\section{References}

1 Wang, Z., Zhang, S., Chen, Y., Zhang, Z. \& Ma, S. Covalent organic frameworks for separation applications. Chem. Soc. Rev. 49, 708-735 (2020).

2 Rodríguez-San-Miguel, D., Montoro, C. \& Zamora, F. Covalent organic framework nanosheets: preparation, properties and applications. Chem. Soc. Rev. 49, 2291-2302 (2020).

3 Song, Y., Sun, Q., Aguila, B. \& Ma, S. Opportunities of Covalent Organic Frameworks for Advanced Applications. Adv. Sci. 6, 1801410 (2019).

$4 \quad$ Geng, K. Y. et al. Covalent Organic Frameworks: Design, Synthesis, and Functions. Chem. Rev. 120, 8814-8933 (2020).

5 Campbell, N. L., Clowes, R., Ritchie, L. K. \& Cooper, A. I. Rapid Microwave Synthesis and Purification of Porous Covalent Organic Frameworks. Chem. Mater. 21, 204-206 (2009).

6 Matsumoto, M. et al. Rapid, Low Temperature Formation of Imine-Linked Covalent Organic Frameworks Catalyzed by Metal Triflates. J. Am. Chem. Soc. 139, 4999-5002 (2017).

7 Zhu, D. et al. Rapid, Ambient Temperature Synthesis of Imine Covalent Organic Frameworks Catalyzed by Transition-Metal Nitrates. Chem. Mater. 33, 3394-3400 (2021).

8 Kandambeth, S. et al. Selective Molecular Sieving in Self-Standing Porous Covalent-Organic-Framework Membranes. Adv. Mater. 29, 1603945 (2017).

9 Karak, S. et al. Constructing Ultraporous Covalent Organic Frameworks in Seconds via an Organic Terracotta Process. J. Am. Chem. Soc. 139, 1856-1862 (2017).

10 Karak, S., Kumar, S., Pachfule, P. \& Banerjee, R. Porosity Prediction through Hydrogen Bonding in Covalent Organic Frameworks. J. Am. Chem. Soc. 140, 5138-5145 (2018).

11 Biswal, B. P. et al. Mechanochemical Synthesis of Chemically Stable Isoreticular Covalent Organic Frameworks. J. Am. Chem. Soc. 135, 5328-5331 (2013).

12 Das, G., Shinde, D. B., Kandambeth, S., Biswal, B. P. \& Banerjee, R. Mechanosynthesis of imine, $\beta$-ketoenamine, and hydrogenbonded imine-linked covalent organic frameworks using liquid-assisted grinding. Chem. Commun. 50, 12615-12618 (2014).

13 Emmerling, S. T. et al. In Situ Monitoring of Mechanochemical Covalent Organic Framework Formation Reveals Templating Effect of Liquid Additive. Chem 7, 1639-1652(2021).

14 Feriante, C. H. et al. Rapid Synthesis of High Surface Area Imine-Linked 2D Covalent Organic Frameworks by Avoiding Pore Collapse During Isolation. Adv. Mater. 32, 1905776 (2019).

15 Sick, T. et al. Switching on and off Interlayer Correlations and Porosity in 2D Covalent Organic Frameworks. J. Am. Chem. Soc. 141, 12570-12581 (2019).

16 Thote, J. et al. Constructing covalent organic frameworks in water via dynamic covalent bonding. IUCrJ. 3, 402-407 (2016).

17 Lu, J. et al. Large-scale synthesis of azine-linked covalent organic frameworks in water and promoted by water. New J. Chem. 43, 6116-6120 (2019).

18 Martín-Illán, J. Á. et al. Green synthesis of imine-based covalent organic frameworks in water. Chem. Commun. 56, 67046707 (2020).

19 Suslick, K. S. Sonochemistry. Science 247, 1439-1445 (1990).

20 Yang, S. T., Kim, J., Cho, H. Y., Kim, S. \& Ahn, W. S. Facile synthesis of covalent organic frameworks COF-1 and COF-5 by 
sonochemical method. RSC Adv. 2, 10179 (2012).

21 Yoo, J. et al. In Situ Synthesis of Covalent Organic Frameworks (COFs) on Carbon Nanotubes and Graphenes by Sonochemical Reaction for $\mathrm{CO}_{2}$ Adsorbents. Chem. Lett. 44, 560-562 (2015).

$22 \mathrm{Xu}, \mathrm{H} ., \mathrm{Gao}, \mathrm{J}$. \& Jiang, D. L. Stable, crystalline, porous, covalent organic frameworks as a platform for chiral organocatalysts. Nat. Chem. 7, 905-912 (2015).

23 Zhi, Y. F. et al. Covalent organic frameworks as metal-free heterogeneous photocatalysts for organic transformations. J. Mater. Chem. A 5, 22933-22938 (2017).

24 Nguyen, H. L. et al. A Porous Covalent Organic Framework with Voided Square Grid Topology for Atmospheric Water Harvesting. J. Am. Chem. Soc. 142, 2218-2221 (2020).

25 Bai, L. Y., Gao, Q. \& Zhao, Y. L. Two fully conjugated covalent organic frameworks as anode materials for lithium ion batteries. J. Mater. Chem. A 4, 14106-14110 (2016).

26 Ascherl, L. et al. Solvatochromic covalent organic frameworks. Nat. Commun. 9, (2018).

27 EL-Mahdy, A. F. M. et al. Strategic design of triphenylamine- and triphenyltriazine-based two-dimensional covalent organic frameworks for $\mathrm{CO}_{2}$ uptake and energy storage. J. Mater. Chem. A 6, 19532-19541 (2018).

28 Auras, F. et al. Synchronized Offset Stacking: A Concept for Growing Large-Domain and Highly Crystalline 2D Covalent Organic Frameworks. J. Am. Chem. Soc. 138, 16703-16710 (2016).

29 de la Peña Ruigómez, A. et al. Direct On-Surface Patterning of a Crystalline Laminar Covalent Organic Framework Synthesized at Room Temperature. Chem. Eur. J. 21, 10666-10670 (2015).

30 Wang, P. et al. Exceptional Iodine Capture in 2D Covalent Organic Frameworks. Adv. Mater. 30, 1801991 (2018).

31 Zhu, D. \& Verduzco, R. Ultralow Surface Tension Solvents Enable Facile COF Activation with Reduced Pore Collapse. ACS Appl. Mater. Interfaces 12, 33121-33127 (2020).

32 Mullangi, D. et al. Highly Stable COF-Supported $\mathrm{Co} / \mathrm{Co}(\mathrm{OH})_{2}$ Nanoparticles Heterogeneous Catalyst for Reduction of Nitrile/Nitro Compounds under Mild Conditions. Small 14, 1801233 (2018).

33 Bai, L. et al. Nanoscale covalent organic frameworks as smart carriers for drug delivery. Chem. Commun. 52, 4128-4131 (2016).

34 Dong, J., Wang, Y., Liu, G., Cheng, Y. \& Zhao, D. Isoreticular covalent organic frameworks for hydrocarbon uptake and separation: the important role of monomer planarity. CrystEngComm 19, 4899-4904 (2017).

35 Meng, Y., Lin, G., Ding, H., Liao, H. \& Wang, C. Impregnation of sulfur into a 2D pyrene-based covalent organic framework for high-rate lithium-sulfur batteries. J. Mater. Chem. A 6, 17186-17191 (2018).

$36 \mathrm{Li}$, X. et al. Molecular Engineering of Bandgaps in Covalent Organic Frameworks. Chem. Mater. 30, 5743-5749 (2018).

37 Shan, Z. et al. Dynamic Transformation between Covalent Organic Frameworks and Discrete Organic Cages. J. Am. Chem. Soc. 142, 21279-21284 (2020).

38 Romero Muñiz, I. et al. Unveiling the Local Structure of Palladium Loaded into Imine-Linked Layered Covalent Organic Frameworks for Cross-Coupling Catalysis. Angew. Chem. Int. Ed. 59, 13013-13020 (2020).

39 Garzón Tovar, L. et al. A MOF@COF Composite with Enhanced Uptake through Interfacial Pore Generation. Angew. Chem. Int. Ed. 58, 9512-9516 (2019).

$40 \quad$ Yang, J. et al. Protonated imine-linked covalent organic frameworks for photocatalytic hydrogen evolution. Angew. Chem. Int. Ed. DOI: 10.1002/anie.202104870 (2021).

41 Nalesso, S., Bussemaker, M. J., Sear, R. P., Hodnett, M. \& Lee, J. A review on possible mechanisms of sonocrystallisation in solution. Ultrason. Sonochem. 57, 125-138 (2019).

42 Smith, B. J., Overholts, A. C., Hwang, N. \& Dichtel, W. R. Insight into the crystallization of amorphous imine-linked polymer networks to 2D covalent organic frameworks. Chem. Commun. 52, 3690-3693 (2016).

$43 \mathrm{Li}, \mathrm{H}$. et al. Nucleation and Growth of Covalent Organic Frameworks from Solution: The Example of COF-5. J. Am. Chem. Soc. 139, 16310-16318 (2017)

44 Stewart, D. et al. Stable and ordered amide frameworks synthesised under reversible conditions which facilitate error checking. Nat. Commun. 8, 1102 (2017).

45 Zhang, J., Han, X., Wu, X., Liu, Y. \& Cui, Y. Multivariate Chiral Covalent Organic Frameworks with Controlled Crystallinity and Stability for Asymmetric Catalysis. J. Am. Chem. Soc. 139, 8277-8285 (2017).

46 Lan, Y. et al. Materials genomics methods for high-throughput construction of COFs and targeted synthesis. Nat. Commun. 9 (2018).

47 Wang, X. et al. Sulfone-containing covalent organic frameworks for photocatalytic hydrogen evolution from water. Nat. Chem. 10, 1180-1189 (2018).

48 Aitchison, C. M. et al. Photocatalytic proton reduction by a computationally identified molecular hydrogen-bonded framework. J. Mater. Chem. A 8, 7158-7170 (2020).

49 Vyas, V. S. et al. A tunable azine covalent organic framework platform for visible light induced hydrogen generation. Nat. Commun. 6 (2015).

50 Bai, Y. et al. Accelerated Discovery of Organic Polymer Photocatalysts for Hydrogen Evolution from Water through the Integration of Experiment and Theory. J. Am. Chem. Soc. 141, 9063-9071 (2019). 


\section{Methods}

Synthesis of sonoCOFs via the sonochemical method. In typical synthesis, the amine and aldehyde monomers are weighed into a $4 \mathrm{~mL}$ vial and aqueous acetic acid $(2 \mathrm{~mL})$ is then added. The mixture is sonicated using a $3 \mathrm{~mm}$ diameter microtip probe driven by a $550 \mathrm{~W}$ Branson Sonifier SFX550 cell disrupter running at $20 \mathrm{kHz}$ in continuous mode and at $50 \%$ power for 60 min. The resulting solids are washed in sequence with acetone, dichloromethane, and methanol, followed by a $24 \mathrm{~h}$ Soxhlet extraction with methanol. The sample is then dried under high vacuum for $24 \mathrm{~h}$. For controls with organic solvents, the aqueous acid phase was replaced by the mixture of 1,4-dioxane $(1 \mathrm{~mL}) /$ mesitylene $(1 \mathrm{~mL}) / 6 \mathrm{M} \mathrm{AcOH}(0.2 \mathrm{~mL})$ or $\mathrm{n}-\mathrm{BuOH}(1 \mathrm{~mL}) / \mathrm{o}-\mathrm{DCB}(1 \mathrm{~mL}) / 6 \mathrm{M} \mathrm{AcOH}(0.2 \mathrm{~mL})$.

\section{Data availability}

All data supporting the finding of this study are available within this article and its Supplementary Information. The experimental procedures and characterization of all COFs are provided in the Supplementary Information.

\section{Acknowledgements}

The authors acknowledge funding from the Leverhulme Trust via the Leverhulme Research Centre for Functional Materials Design and the Engineering and Physical Sciences Research Council (EPSRC). P.Y., H.C., L.L. and B.L. thank the China Scholarship Council for a PhD studentship. We thank the Materials Innovation Factory (MIF) team for help with instrument training. We thank Molly Lightowler of Stockholm University for testing the feasibility of using 3D electron diffraction to solve the COF structures. The TEM analysis was performed in the Albert Crewe Centre for Electron Microscopy, a University of Liverpool Shared Research Facility.

\section{Author contributions}

W.Z. synthesized and characterized the materials, performed photocatalytic experiments and analyzed the photocatalysis results with H.Y. P.Y. performed the SEM, TCSPC and FT-IR measurements. H.Y. performed the UV measurements. R.C. performed TGA measurements. A.M.J. and W.Z. performed gas adsorption measurements. H.C. and L.L. performed photoelectrochemical measurements. M.B. and N.D.B. performed TEM measurements. W.Z. conceived the modelling strategy. B.L. and Z.P. provided useful advice in the structural simulation of COFs. Y.W. and J.W.W. conceived the project. A.I.C., Y.W. and J.W.W directed the research. Data were interpreted by all authors and the manuscript was prepared by A.I.C., Y.W., J.W.W. and W.Z.

\section{Competing interests}

The authors declare no competing interests. 